\title{
Technical Analysis and Design of New Mobile OA System
}

\author{
Lanyu Zhang ${ }^{1}$ \\ (College of Mathematics, Jilin University, Changchun, Jilin Province, 130012)
}

Key words: OA system, cloud computing, smart phone

Abstract: This paper is a thorough study into the design and construction of office automation and its application in Android system, and it also puts forward a solution for the system challenges that exist in the market currently, in an effort to improve the existing OA system, to enable it to function better and to provide better service for modernization construction.

\section{Preface}

By OA system it means to fulfil automated and paperless office work through advanced technology. Its birth has brought about a brand new way of working for enterprises and public institutions and soon become an indispensible part of these organizations. Although the OA technology is well developed now, there are still quite a lot of defects in it. The traditional OA system has many restrictions on the facilities and locations of the office, which to a great extent restricts the freedom of those people that work out of the office, and the system development and upgrade are a great burden for small and medium sized firms. However, with the fast growth of cloud computing technology and smart phone, cloud OA and mobile OA come into shape which bring about sound solutions to the abovementioned problems. Focusing on these problems, this paper integrates the above 2 features and designs a mobile computing OA system.

\section{Framing Analysis of Mobile Cloud OA System}

We put forward the concept of mobile cloud OA in an effort to overcome the defects of traditional OA system. With the smart phone technology and cloud computing integrating with and complementing each other, it's able to overcome many innate defects of cloud OA and mobile OA and puts forward the mobile cloud OA system that can replace the existing traditional one. The OA system designed in this paper sets its OA server on the cloud which provides general OA service plus common cloud service like cloud saving and cloud application. An intermediate server is also set up under the cloud which functions in providing OA service for users without affecting the work of the original OA system. Users that $\log$ in to the system through PC are able to visit OA server directly while those that log in through mobile terminals visit it through the intermediary server. In this way it's able to guarantee that the PC terminal and mobile terminals are synchronized thorough cloud service. ${ }^{[1]}$

The procedure to deliver it is shown below in diagram 2.1.1 


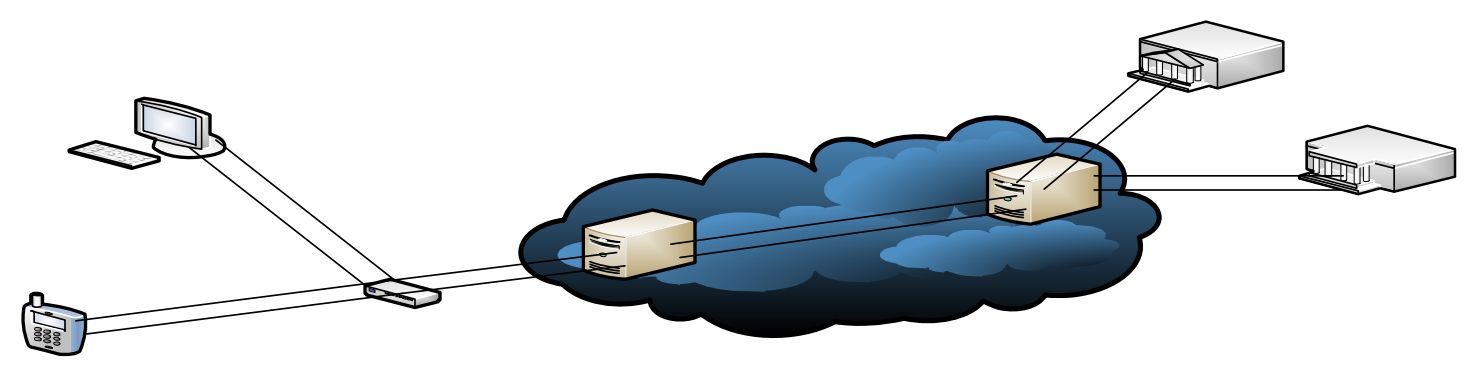

Diagram 2.1.1 Mobile Cloud OA Framework

\section{Module Design}

\section{Information Publish and Management}

Information Publish and Management platform covers functions like publishing info, public service and homepages of organizations. Among them, the info publish section allows the users to browse the latest campus documents, campus notices, teaching info, meeting info, academic info and student scores released by schools. In this way users on all levels are able to access the latest news of schools in a convenient and quick way.

\section{Document Management and Exchange System}

Documents edited by a department are released for users in other departments to read, to sign for and to download. When the user clicks the document, it would be displayed on the PC or mobile phones of the user with Office software in the server to open it, and in this way the user is able to read and fill in the document in the server. After the user has signed for the document, it can be sent back to the publisher directly. If the document is downloaded, the user needs to install Office software in order to open and fill in it.

\section{Personal Office System}

Personal Office System includes features like personalized interface setting, address book, memo pad and wifi log-in etc. This helps to fulfil a personalized office automation system and users can customize their personal info like their own work arrangement and address list based on actual needs.

\section{Mobile Platform}

Due to big difference in the sizes of PC and mobile phone screens, it's difficult to fulfil a convenient B/S structure used on smart phones. Thus for the purpose of convenient browsing on the phone, C/S structure should be used, which means to visit the office system through the user's end installed on mobile phones.

\section{Design of Intermediary Server}

The core design of this system lies in the intermediary server, which links the mobile devices to the OA server. It consists of linking modules, proxy modules, transitional modules and management modules. ${ }^{[2]}$

\section{Overall System Implementation}

There are two ways for system implementation, both online and offline.

\section{Offline:}

While the user is visiting the office system, its data would be transmitted to the user's device which is then processed with software and hardware of the device. After this the data is sent back to the server. 


\section{Online:}

While the user is requesting something, the server receives the request and extracts some data, but it doesn't return the data to user's device instead it saves the data to the server after it processes the requested data on the server with its software and hardware.

\section{Info Publishing and Management Module}

Based on the role of the publisher, the module needs to tell first whether he has the right to send the info. The publisher needs to determine the format in which the info is uploaded, check whether there's any attachment, the department such info goes to and timing, and fill in the content of the info on the publishing page.

Procedure of this module is shown in Diagram 4.2.1:

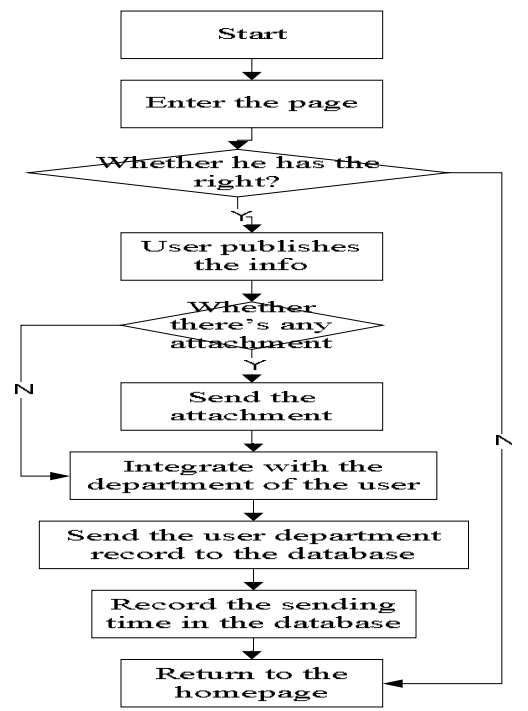

Diagram 4.2.1 Software Flow plan of Info Publishing Module

\section{Documents Management System}

To establish a documents management system, the biggest challenge that we need to tackle with is to save the handwriting. This can be done by adding a control into the system which supports the feature of preserving the inking. This control functions not only in taking correct record of the inking on the handwriting input screen, but also in integrating the electronic stamp file with the targeted document that needs to be recorded, and only in this way it suffices the system. ${ }^{[3]}$

The primary method to establish this control is to record the lateral and longitudinal lengths of the Word or PDF documents as coordinate axis thus form a plane coordinate system attached to the document. When the stylus touches the handwriting input screen, or when the electronic stamp moves below the middle line of the document, its coordinates in the coordinate system are recorded. Then the trace of the stylus or the electronic stamp is saved as JPG and put into the Word or PDF documents, which is shown in Diagram 4.3.2 


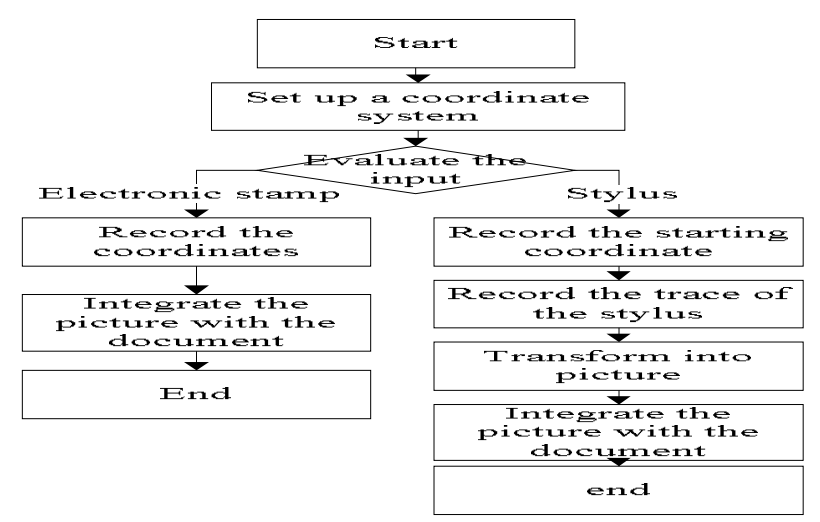

Diagram 4.3.2 Software Flow Plan for Document Transmission

Simulated web uploading method based on HTTP transfer protocol needs to seal the uploaded document in fixed HTTP format.

\section{Interaction Function}

By interaction function it means that the message is sent to server's database by the user's device. When the targeted person goes online he can download the messages from other users. Basically the message function is to upload the text to the data server.

\section{Linking Service}

OA platform is linked to external service which expands the system's functions to any other field. Various departments can also link their servers to the office system of the schools. ${ }^{[4]}$

Part of these codes goes like this

URL OurUrIInfor = new URL(FilePath);

int TransPort $=$ OurUrlInfor.getPort ()$==-1$ ? 80 : OurUrlInfor.getPort () ;

Socket OurPrviateSocket $=$ new

Transmission process from collection by the user's device to output by the server

OutputStream TheStreamsFromFile = OurPrviateSocket.getOutputStream();

\section{User's Mobile Phone Terminal}

If the user logs in to the OA through external networks on a mobile phone, it first of all goes through the OA to set up the connection. After this the data is exchanged through the API port reserved in the system, which is shown in Diagram 4.6.2

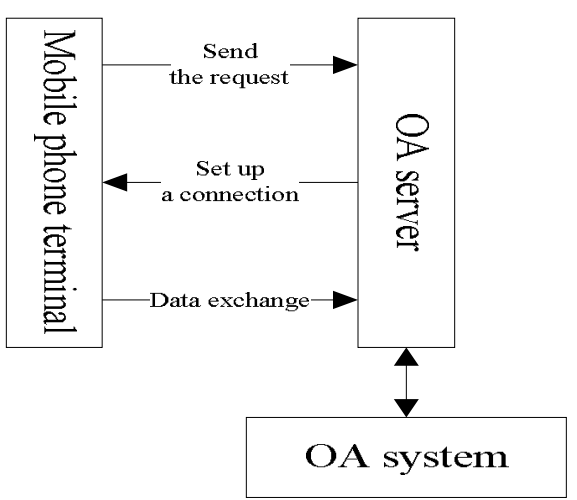

Diagram 4.6.2 Flow chart for mobile phone office system 
1. The mobile app sends URL of certain request to a designated OA server with a HTTP protocol, and the codes are shown below

$$
\begin{aligned}
& \text { String FilePath }=\text { "...";//Access path of the server }(\text { Acrtion }) \\
& \text { URL OurUrlInfor = new URL (FilePath); } \\
& \text { //enabling it to link and start linking to the target } \\
& \text { HttpURLConnection Connection= (HttpURLConnection) }
\end{aligned}
$$

2. The mobile phone sets up an exclusive virtual network after it's connected through the OA. Data is exchanged with the OA through an API port and then the user's device would display the data transmitted from there.

3. The server end would receive this request through the correspondent Action. Since in the default setting of all web servers this information is responded through HTML protocol, it's essential to restructure the info in the format of XML. ${ }^{[5]}$

\section{Conclusion}

With the development of technology, OA improves the working efficiency of enterprises and government departments to a great extent, however traditional OA has specific requirements on hardware and space which are difficult to realize for small and medium sized enterprises and public institutions. Meanwhile this is very inconvenient people that work on a mobile base. With fast development of cloud computing and smart phone, the mobile cloud OA will inevitably come into being.

This paper looks into the defects of cloud OA and mobile OA and put forward the mobile cloud OA structure, in which 3 important components are established including OA server, user's mobile terminal and intermediary server.

The system designed in this paper does not affect the original OA. No extra OA server needs to be set up and it only needs to add an intermediary server into the original system, which is very convenient. The system solves the security concern, instable network and excessive data transmission of mobile working; meanwhile it also guarantees the adaptability of mobile system interface, which is a very practical approach for the users.

\section{Reference}

1. Zhongwei Li, Xiaotong Zhou, Dandan Chen, From Java ABC to Proficiency, Tsinghua University Press, 2010

2. Xiaofeng Cui, Xiaohui Li, Delphi7 Distributed App System Development, Beijing, China Railway Publishing House

3. Zhilong Yu and others, Collection of Android SDK Developments, 2nd edition, Posts \& Telecom Press, 2010

4. Shijiang Wang, Zhilong Yu, Lixun Chen, Mingjie Zheng, Collection of Google Android SDK, Posts \& Telecom Press, 2010

5. Fengsheng Yang, Uncovering Android App Development, Machine Engineering Press, 2010 